\title{
ON-LiNE OPTIMIZATION METHOD FOR ENERGY EFFICIENT PUMP-STORAGE OPERATION WITH INTEGRATED FILLING Time CONSTRAINT
}

\author{
Thomas Hieninger, Florian Goppelt and Ronald Schmidt-Vollus \\ Nuremberg Campus of Technology, TH Georg Simon Ohm, Nuremberg, \\ Germany
}

\begin{abstract}
This contribution presents a Nelder-Mead based on-line optimization algorithm for centrifugal pumps to decrease the energy demand for filling tasks during operation. This method works with power and filling level data, which adapts the rotational speed to the current filling level. During optimization, rotational speed constraints have to be regarded to fulfill a steadily filling within a maximum permitted time. To develop and to test the algorithm, a model of a pump system is used, which is implemented in Matlab/Simulink. The tuning method achieves energy savings at the permitted filling time.
\end{abstract}

\section{KEYWORDS}

Online-Optimization, Centrifugal Pump, Energy Efficiency, Fluid Storage

\section{INTRODUCTION}

A primary application for electric motors with a share of 19 percent is the operation of centrifugal pumps. This widespread distribution leads to nine percent of the worldwide electrical energy demand [1]. An essential task of pumps is to fill storages, which may occur in water distribution systems or industrial plants.

Two different effects have to be focused on to increase the energy efficiency of filling processes. First of all, the best efficiency point is not necessarily the most efficient operating point for filling storages. The speed should be lowered to avoid dynamic losses, whereas the resulting part load leads to lower efficiencies. Secondly, the operating point moves depending on the increasing head during the filling process. Therefore, a clearly defined solution is not available, which can be solved by employing an optimization algorithm (OA).

An approach with a volume flow meter is described in [2]. Another way is to adapt the rotational speed to the current filling level to avoid additional losses and costs due to the flow meter [3]. The authors in [4] showed a method and the potential energy savings employing a filling control strategy. The strategies in [3] and [4] leads to the additional issue, that the filling time of an energetic optimized storage system can be significantly extended. Thus, a set-point for maximum filling time may be necessary, depending on the application. 
It is also a problem that the real behavior can vary from the behavior according to the data-sheet given pump characteristics and the calculated plant characteristic. This deviation leads to the approach that the frequency converter supported power estimation or measured power values in combination with an already existing sensor as input information for an on-line tuning strategy leads to exact results. In this way, the algorithm tunes the real system behavior to the most efficient operation (Figure 1). An advanced Nelder-Mead (NM) algorithm [5] is chosen and modified by a noise signal to improve the convergence behavior to adjust the speed.

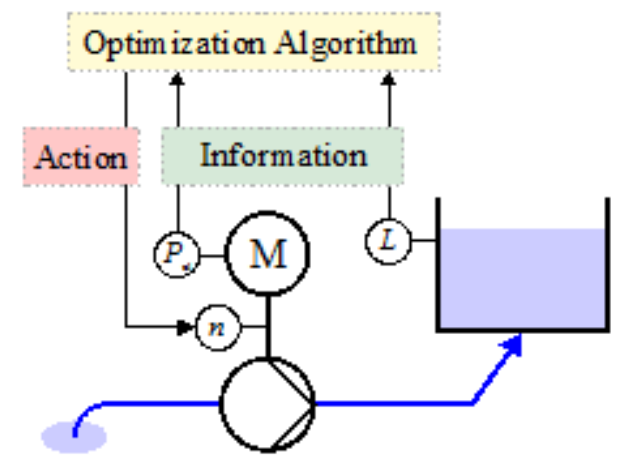

Figure 1. Pump system with a source, pipeline, storage and electric motor including the needed information for the optimization algorithm.

A model of a pump system with a pipeline and storage is realized in Matlab/Simulink, which is presented in this contribution, to develop the algorithm. Furthermore, two kinds of optimization constraints, which are implemented in the system, are displayed. These constraints ensure a maximum filling time and a steady filling process.

\section{Model of Centrifugal Pump and Fluid Storage System}

The regarded system (Figure 1) consists of a variable speed driven centrifugal pump, a pipeline and fluid storage, which is filled from below. This filling from below leads to an increasing static head during filling and therefore to a change in the system characteristics.

This system can be divided into three physical subsystems. The first one is the pumping set, which determines the delivery head and the shaft power for a certain rotational speed and flow rate. The second part uses the pump head to calculate the dynamic head in the pipeline subsystem. The third subsystem, the storage subsystem calculates the current storage level utilizing the flow rate, which affects back to the pump system (Figure 2).

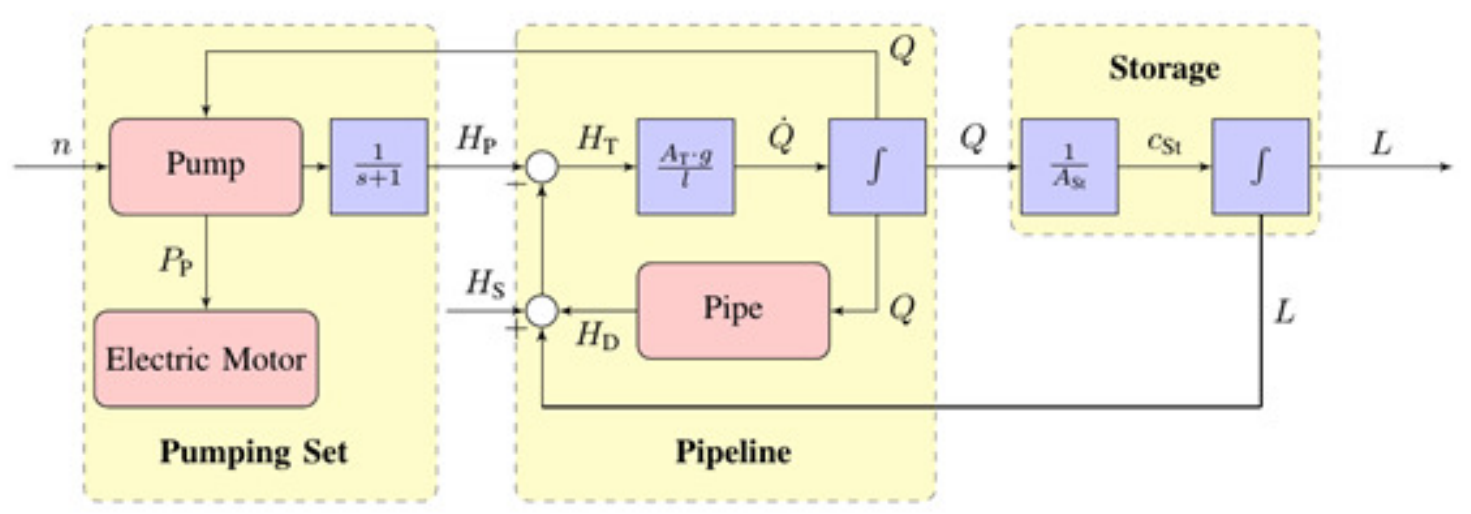

Figure 2. Block diagram of a pump system with pipeline and storage. 


\subsection{Pumping Set}

The characteristic curves of power $P_{\mathrm{P}}$ describe the pump behavior and pump head $H_{\mathrm{P}}$ as functions of the flow rate $Q$ (Figure 3). Employing the affinity laws the rotational speed $n$ and the reference rotational speed $n_{\mathrm{n}}$ are included in the model. Proper results for $H_{\mathrm{P}}$ and $P_{\mathrm{P}}$, depending on pump series, can only be reached in the closer area of the reference characteristic curve. This effect is caused by varying maximum efficiency peaks at different speeds due to effects like inappropriate incident flow or return flow [6].
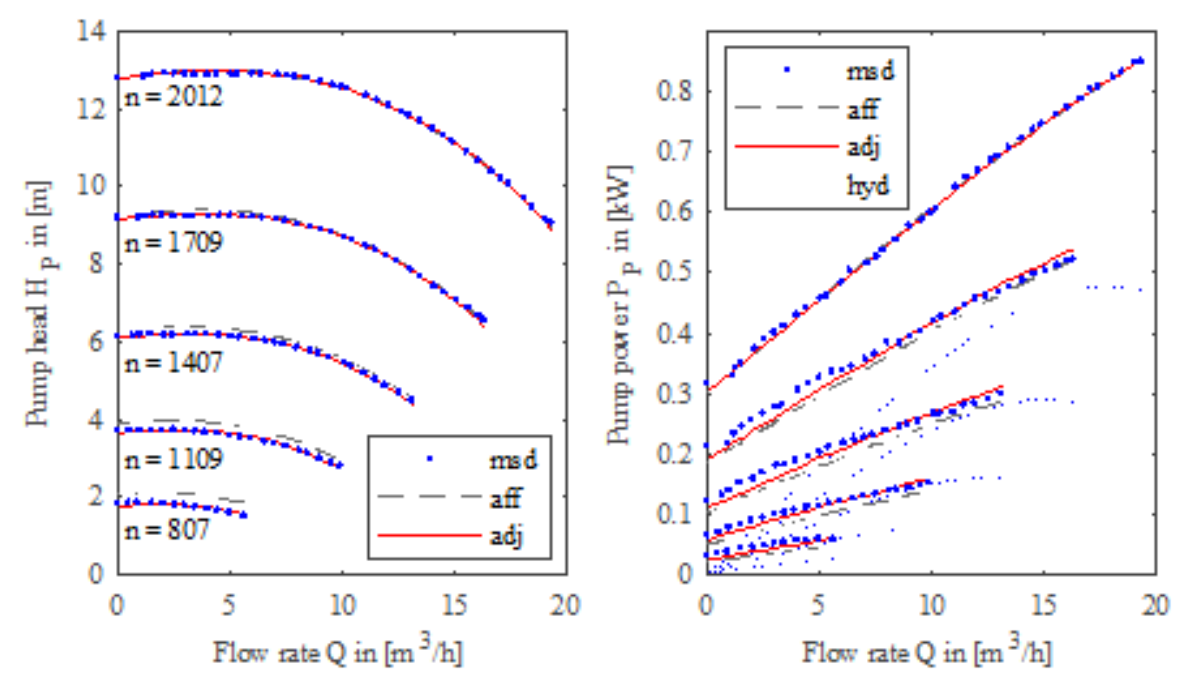

Figure 3. QH- and QP-characteristics of a modeled pump with the measured values (msd), affinity laws (aff), adjusted values (adj) and hydraulic power (hyd).

The reference speed Is $n_{\mathrm{n}}=2012 \mathrm{rpm}$.

The modeled pump KSB Etanorm 050-032-125 characteristics are measured and calculated with the help of polynomial functions of order $j$ with their polynomial coefficients $A_{i}$ and $B_{i}$. Correction parameters $c_{1,2}$ are implemented (Eq. 1) to adapt the head deviations. Considering the differences of $P_{\mathrm{P}}$ the method according to Staufer with the assumption $n_{1} / n_{2}=R e_{1} / R e_{2}$ is adduced (Eq. 2) [7] after [8]. Using this adjusted affinity laws better correlations between measured and modeled results are given (cf. Figure 3).

$$
\begin{gathered}
H_{\mathrm{P}}=\sum_{i=0}^{j}\left(A_{i+1} \cdot Q^{j-i} \cdot\left(\frac{n_{\mathrm{n}}}{n}\right)^{j-i-2}\right)+c_{1} \cdot n+c_{2} \\
P_{\mathrm{P}}=\sum_{i=0}^{j}\left(B_{i+1} \cdot Q^{j-i} \cdot\left(\frac{n_{\mathrm{n}}}{n}\right)^{j-i-3}\right)
\end{gathered}
$$

\subsection{Pipeline and Storage System}

The pipeline ( $\mathrm{PT}_{1}$-system) calculates a one-dimensional flow-the static head is set to $H_{S}=0$ due to time independence; $g$ describes the gravity, $A_{\mathrm{T}}$ the cross-sectional area of the pipe, $l$ the pipe length and $k$ (Eq. 4) the dynamic pipe resistance. The storage (I-system) consists of its cross- 
sectional area $A_{\mathrm{St}}$ and the filling level $L$. Both systems combined can be described with the transfer function (Eq. 3) according to [3].

$$
\begin{gathered}
\ddot{Q}+\frac{2 \cdot A_{T} \cdot g \cdot k}{l} \dot{Q} Q+\frac{A_{\mathrm{T}} \cdot g}{l \cdot A_{\mathrm{St}}}=\frac{A_{T} \cdot g}{l}\left(\dot{H}_{\mathrm{P}}-\dot{L}\right), Q \leq 0 \\
k=\frac{1}{2 \cdot A_{\mathrm{T}}^{2} \cdot g} \cdot\left(\lambda \cdot \frac{l \sqrt{\pi}}{2 \sqrt{A_{\mathrm{T}}}}+\zeta\right)
\end{gathered}
$$

Here $\zeta$ terms the loss coefficient and $\lambda$ the friction factor, which is divided into three ranges depending on the Reynolds Number Re (Eq. 5). In the turbulent flow regime, the factor is determined by the approximation after [9], whereby $r$ describes the tube roughness. In the laminar regime, the Hagen-Poiseuille law and in the transition area $(2320 \leq \operatorname{Re} \leq 3000)$ quadratic interpolation are applied.

$$
\lambda= \begin{cases}64 / \operatorname{Re} & \text { for } R e<2320 \\ 1 /\left(-1.8 \ln \left(\frac{6.9}{\operatorname{Re}}+\left(\frac{0.5 \sqrt{\pi} r}{3700 \sqrt{A_{\mathrm{T}}}}\right)^{10 / 9}\right)\right)^{2} & \text { for } R e>3000\end{cases}
$$

\subsection{Model Verification}

To verify the static behavior the correlations of the measured values and the simulated values are shown using the impeller diameter $D$, the dimensionless head $\Phi$ (Eq. 6) and power $\Xi$ (Eq. 7) in Figure . For both characteristics, good correlations are reached (slope of the mean relationship $(\rho \approx 1)$. At lower speeds $(n \approx 800 \mathrm{rpm})$ the modeled and measured power are deviating.

$$
\begin{aligned}
& \Phi=H_{\mathrm{P}} /\left(n^{2} \cdot D^{2}\right) \\
& \Xi=P_{\mathrm{P}} /\left(n^{3} \cdot D^{5}\right)
\end{aligned}
$$


Figure 4. Correlation of simulated and measured dimensionless head and dimensionless power at different speeds (cf. Figure 3).

The model is also tested to its slow transient behavior. Several step responses are recorded for this purpose (Figure 5). The measured speeds are implemented in Simulink. Thus, the simulated speeds are the same. Good results for system gains can be achieved in the range of the reference 
speed $n_{\mathrm{n}}=2012 \mathrm{rpm}$. At lower speeds, the deviations for head and flow rate are increasing. The overshoot for $H_{\mathrm{P}}$ in the model is higher than the measured one, which causes a higher overshoot for $Q$. This effect alone does not explain the complete simulated overshoot for $Q$. For the sake of a robust simulation further model fitting is not done.
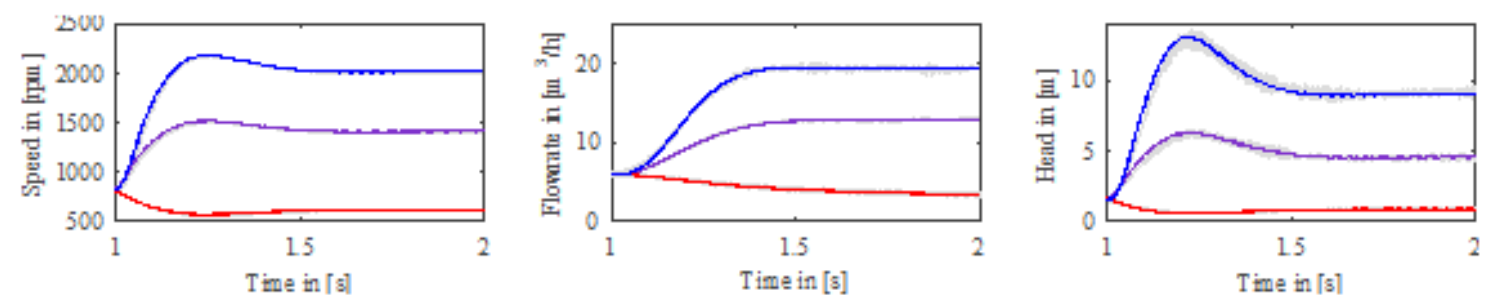

Figure 5. Simulated (grey) and measured step responses for flow rate and pump head for rotational speed set points: $n=600 \rightarrow 800 ; n=800 \rightarrow 1400 ; n=800 \rightarrow 2000$.

\section{OPTIMIZATION WITH SYSTEM INTEGRATED CONSTRAINTS}

To satisfy maximum time limits and also to avoid an unsteady filling process rotational speed constraint have to be implemented. The relationship between parameterization of the speed control, which is guided by the current filling level and the maximum time respectively an unsteady filling process $\left(H_{\mathrm{P}} \leq L\right)$ is not precisely defined. Because of that lack of knowledge, the constraints can't be directly integrated into the OA. They are calculated through the time force control (TFC) and level force control (LFC).

The realization of the tuning program consists of several subroutines and is shown in Figure 6. At the beginning, a function for predicting a filling level $L_{\mathrm{c}}$ has to be adapted. After that, the system is operating using the given starting values $\overrightarrow{a_{0}}$. The NM can begin when the best result of $w_{0}$ is running without TFC. The operation is continuously checked by the subroutines LFC and TFC.

\subsection{Optimization}

The minimization of the energetic energy demand per filling is realized by adapting parameters $a_{i}$, which describe the coherence between speed and filling level and the static head:

$$
n_{\mathrm{opt}}=a_{1}+a_{2} \cdot\left(L+H_{\mathrm{S}}\right)^{a_{3}}
$$

For finding the values of $a_{i}$, the NM algorithm is modified to improve the optimization. Firstly, if the current target value $w$ is smaller than $w<0.7 \cdot w_{0, \text { min }}$, the OA creates new starting values $a_{0}$ around the new best point to better examine this area. Secondly, to improve the dynamic behavior of the algorithm, the calculated parameters for $\vec{a}$ are varied with a noise signal $s$ (Eq. 9). NM uses the centroid vector $\overrightarrow{a_{\mathrm{c}}}$ of $n+1$ values for an $n$-dimensional problem in combination with a chosen action $\vec{x}$. Because of the decreasing influence of the NM-action due to nearing values to the centroid, the impact of the noise is fading away.

$$
\vec{a}=\overrightarrow{a_{\mathrm{c}}}+\vec{x} \cdot s \text { with } \vec{a}=\left(\begin{array}{l}
a_{1} \\
a_{2} \\
a_{3}
\end{array}\right)
$$


The target value $w$ in this contribution is the used filling energy $E$, which is calculated by integrating $P_{\mathrm{P}}$ (Eq. 10). In case of using LFC a penalty value $E^{+}$is added to the target value. For using TFC no additional penalty is necessary through an increase of power at higher speeds. The OA is terminated when $b$ in Eq. 11 is smaller than a given termination tolerance ( $w_{j}$ are the $n$ best target values and $w_{\mathrm{c}}$ is the centroid of them).

$$
\begin{gathered}
w=E^{+}+\int_{t=0}^{t=\max } P_{\mathrm{P}} \cdot d t \\
b=\sqrt{\sum_{j=1}^{n} \frac{\left(w_{j}-w_{\mathrm{c}}\right)^{2}}{n}}
\end{gathered}
$$

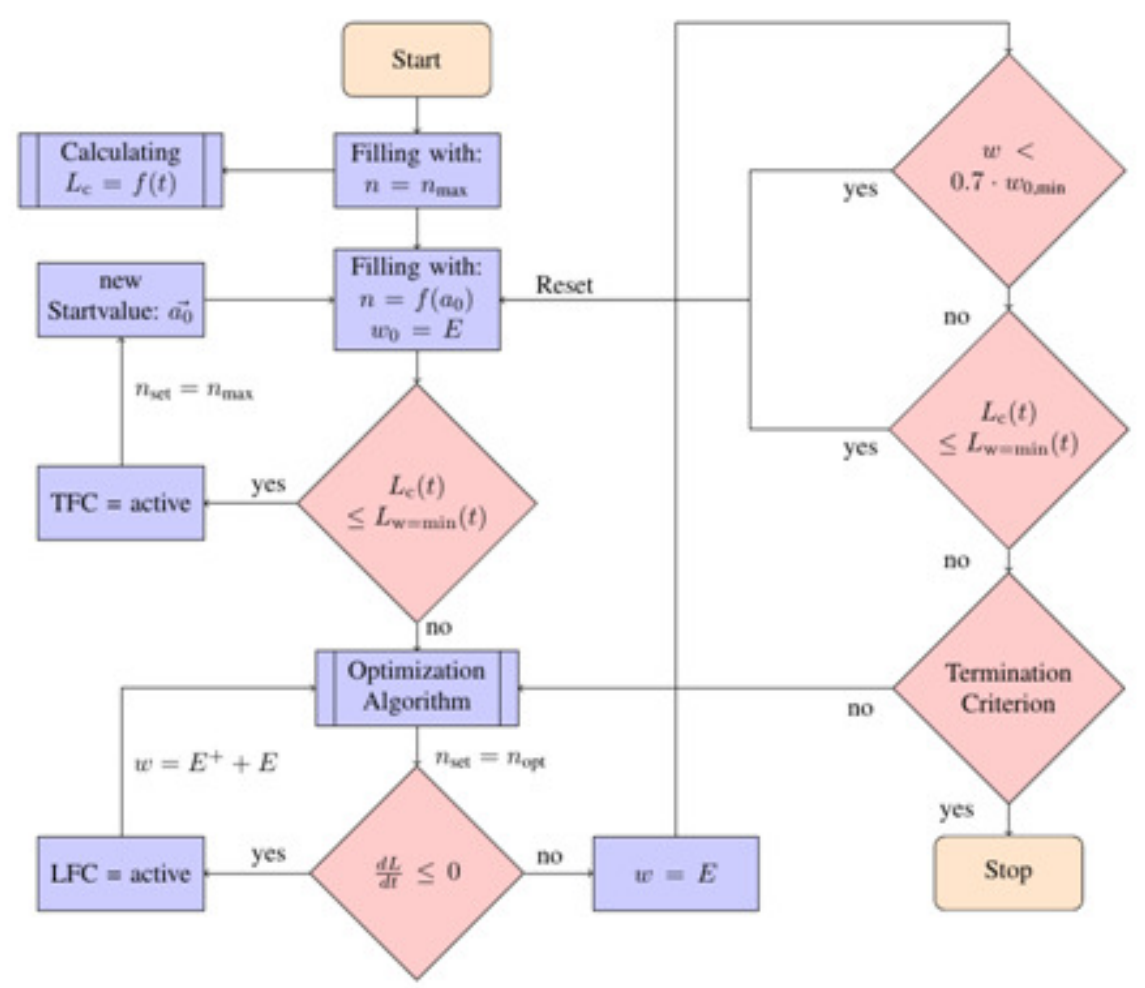

Figure 6. Flow chart for the tuning program.

\subsection{Built-In Constraints}

The TFC checks to see, which predicted filling level $L_{c}$ can be achieved during a maximum filling time $t_{\max }$. Therefore, the pump is operating with maximum speed at the first filling to the given filling level $L$ to determine the shortest possible filling time $t_{\mathrm{n}=\max }$. In the next step, the TFC can be applied by checking Eq. 12 at the current filling time $t$ :

$$
n_{\mathrm{set}}=\left\{\begin{array}{ll}
n_{\mathrm{opt}} & \text { for } L_{\mathrm{c}}>L \\
n_{\max } & \text { for } L_{\mathrm{c}} \leq L
\end{array} \text { with } L_{\mathrm{c}}=\frac{\Delta L}{t_{\mathrm{n}=\max }} \cdot\left(t_{\max }-t\right)\right.
$$


The LFC consists of a discrete ID-controller transfer function. An impulse with amplitude $\sigma=1$ is given to the controller if $d L / d t \leq 0$, which leads to a constant increase of speed. An exemplary behavior of both constraints is shown in Figure 7.


Figure 7. Filling with maximum filling time $t_{\max }=4000 \mathrm{~s}$ using LFC and TFC.

\section{BEHAVIOR AND ANALYSIS OF THE OPTIMIZATION ALgORITHM}

The tuning algorithm is tested five times each for six maximum filling times. With a maximum permitted filling time of $t_{\max }=5000 \mathrm{~s}$ no time constraint is necessary because of $t_{\text {Energy }=\min }<t_{\max }$. For this case and for $t_{\max }=3500 \mathrm{~s}$ the tuning processes are shown in Figures 8 and 9.


Figure 8. Decrease of the target value $w$ and tuning of $a_{1}, a_{2}, a_{3}$ during optimization with maximum filling time $t=3500 \mathrm{~s}$. 

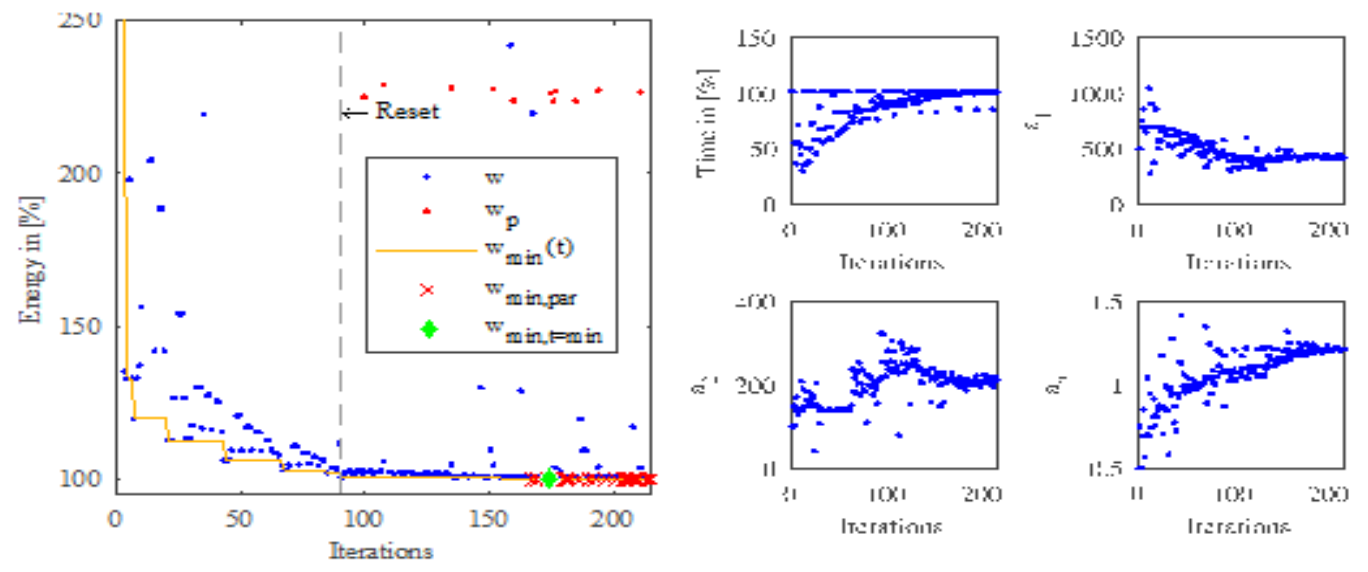

Figure 9. Decrease of the target value $w$ and tuning of $a_{1}, a_{2}, a_{3}$ during optimization with maximum filling time $t=5000 \mathrm{~s}$.

It can be seen, that the target values $w$ respectively $w_{\mathrm{p}}$ (with additional penalty $E^{+}$) are decreasing to a minimum during the tuning process. The trends of $w_{\min }$ show the minimum detected amounts at the current optimization step. Several best points are found before the termination criterion cancels the optimization. This Pareto optimality is shown with $w_{\text {min,par }}$, whereby $w_{\text {min,t=min }}$ shows minimum filling energy in combination with minimum filling time. The reset points are occurring due to $w=0.7 \cdot w_{0, \min }$.

The final results for all optimizations are shown in Figure 10. The tuned energy $E_{\mathrm{opt}}$ is compared to the energy demand $E_{\mathrm{bf}}$ optimized by Brute Force and $E_{\mathrm{q}}$ using constant flow rate control. Especially at shorter filling times, the percentage $w$ for continuous flow rate rises sharply. The Brute Force method shows that good results are reached using this presented optimization method.
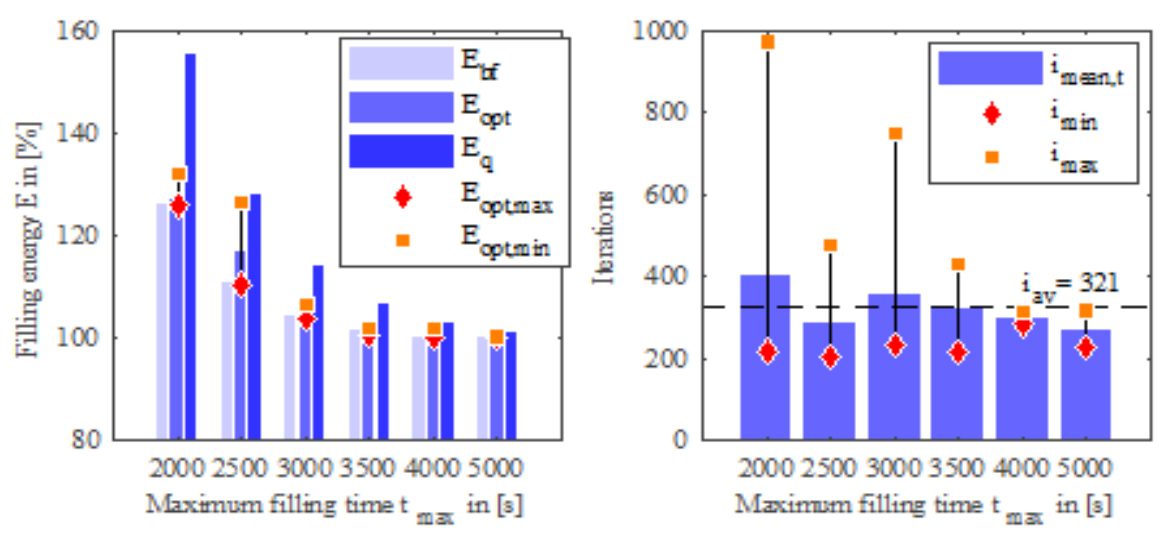

Figure 10. Energy demand referred to its minimum and resulting iterations for five optimizations per fixed maximum time.

The bars for $E_{\text {opt }}$ are showing the mean results for five tuning processes, whereby $E_{\text {opt,max }}$ and $E_{\text {opt,min }}$ are the maximum and minimum found final value. The authors recommend more optimizations per filling time to avoid these local minima. The average number of iterations is given with $i_{\mathrm{av}}=321$. Nevertheless, two times the numbers of iterations $i>700$ are occurring. 


\section{CONCLUSION AND OUTLOOK}

In this contribution, an energetic optimization for centrifugal pumps to fill storages was presented. The requirement for the task is a maximum permitted filling time, which is handled by the time force control. An additional subroutine, the level force control, ensures a steadily filling process.

Using this method, frequency-based power estimation or installed power measurement in combination with and filling level sensor the plant can be tuned on-line during operation without plant characteristics from data-sheets and calculated pipeline resistance. Compared to fillings with a constant flow rate, high savings are possible, especially at shorter filling times.

The presented method for energetic optimization of filling fluid storages with a maximum permitted filling time will be tested in a pump test rig in future work. Additional problems like measurement noise for the power and filling level signals are expected. This difficulty may be managed by implementing proper filter methods. Another possible parameter to adapt the rotational speed is the current filling time, which reduces installation costs by abandoning the level sensor.

\section{ACKNOWLEDGMENTS}

Martin Weber for helping with the development and implementation of the algorithm.

This work was conducted within the project DATAePump, funded by the European Union and EFRE Bayern.

\section{REFERENCES}

[1] Waide \& Brunner, (2011) "Energy-efficiency policy opportunities for electric motor-driven systems".

[2] Lindstedt \& Karvinen, (2006) "Optimal control of pump rotational speed in filling and emptying a reservoir: minimum energy consumption with fixed time", Energy Efficiency, Vol 9, pp 1461-1474.

[3] Hieninger \& Schmidt-Vollus, (2017) "Self-tuning pump operation mode for fluid storages to increase energy efficiency", CCWI - Computing and Control for the Water Industry 2017.

[4] Santos \& Seleghim, (2005) "Optimized strategies for fluid transport and reservoir management", Minerva, Vol 2, pp 91-98.

[5] Zhao, Urosević, Mladenović \& Hansen, (2009) "A restarted and modified simplex search for unconstraint optimization”, Computers \& Operation Research, Vol 36, pp 3263-3271.

[6] Gülich, (2013) Kreiselpumpen, Springer Vieweg.

[7] Staufer, (1925) "Einflüsse auf den Wirkungsgrad von Wasserturbinen”, VDI, pp 415.

[8] Heß, (2010),Aufwertung bei Axialventilatoren - Einfluss von Reynolds-Zahl, Rauheit, Spalt und Betriebspunkt auf Wirkungsgrad und Druckziffer, Dissertation Technische Universität Darmstadt.

[9] Haaland, (1983) "Simple and explicit formulas for the friction factor in turbulent flow", Journal of Fluids Engineering, pp 89-90. 


\section{Authors}

Thomas Hieninger (M. Sc.),

2007-2011: Mechanical Engineering (B.Eng.) at OTH Regensburg

2011-2013: Process Engineering (M.Sc.) at Friedrich Alexander University ErlangenNürnberg since

2014: Research Assistant at Nuremberg Campus of Technology; active in the field of energy efficient control of pump systems

Florian Goppelt (M. Eng.)

2010-2014: Mechatronics (B.Eng.) at TH Nürnberg Georg Simon Ohm

2014-2015: Electronical and Mechatronic Systems (M.Eng.) at TH Nürnberg Georg Simon Ohm Since

2016: Research Assistant at Nuremberg Campus of Technology; active in the field of mathematical modelling, control and observation of pump systems
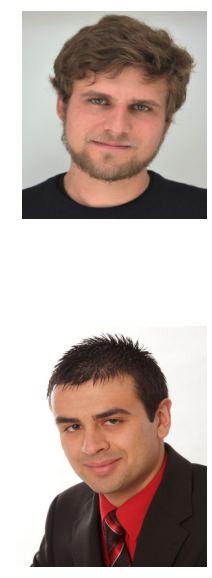

Prof.-Dr.-Ing Ronald Schmidt-Vollus

1991-1997: Education in Chemical Engineering at Friedrich Alexander University Erlangen-Nürnberg (FAU)

1998-2004: Control engineering and process automation, commissioning of process control systems

1994-2006: Development associate for process control systems

2006-2013: Head of Development (process control) ProLeiT AG, Herzogenaurach

2011: Ph.D. Degree (Dr.-Ing) at Friedrich Alexander University Erlangen-Nürnberg (FAU)

Since 2013: Professor for control technologies, Nuremberg Campus of Technology, Technische Hochschule Nürnberg Georg Simon Ohm. 